\title{
Sample matrix influence on microdrop hold-up in single drop microextraction
}

\author{
Nikola Kubasová, Silvia Zichová, Adriána Brisudová, Svetlana Hrouzková \\ Slovak University of Technology in Bratislava, Faculty of Chemical and Food Technology, \\ Institute of Analytical Chemistry, Radlinského 9, 81237 Bratislava, Slovak Republic \\ svetlana.hrouzkova@stuba.sk
}

\begin{abstract}
Single drop microextraction has become a widespread liquid/liquid microextraction technique owing to its simplicity, high preconcentration factor and low consumption of organic solvents in the extraction due to direct introduction of the very low volume of extract into the analytical system. Crucial features ensuring excellent repeatability of single drop microextraction include: solvent volume, solvent type, sample agitation, salts addition, and $\mathrm{pH}$. The influence of sample quality on the microdrop volume and agitation type was studied. Effect of the sample matrix, such as water, acid content samples (orange juice), sample containing alcohol (plum brandy) and protein content sample (milk), on the microdrop hold-up was also investigated. For water analysis, several organic solvents such as chloroform, dichloromethane, tetrachloromethane, tetrachloroethane and chlorobenzene were tested; last three mentioned were suitable for SDME experiments. For milk samples analysis, chlorobenzene microdrop was found to be optimal; advantage of salt addition has been reported. For orange samples, 1:10 dilution was suggested with stable microdrop volumes (toluene) of up to $6 \mu \mathrm{L}$ for lower stirring rates $(100 \mathrm{rpm}$ and $250 \mathrm{rpm}$ ). For alcohol-content samples, the change of alcohol percentage of real-life samples had to be considered. A strong influence of the matrix quality on the microdrop stability has been proven.
\end{abstract}

Keywords: matrix influence, microdrop hold-up, microdrop stability, single drop microextraction

\section{Introduction}

Actual trends in analytes extraction from various food or environmental samples follow green chemistry philosophy taking into account simplification and miniaturisation, especially minimisation of the volume of necessary organic solvents which are potentially harmful and unwanted in large scale in analytical procedures (Hrouzková, 2017). For the liquid-liquid extraction, a set of techniques called liquid-phase microextraction (LPME) has underwent a dramatic increase in a variety of approaches (Andraščíková et al., 2015) using only microliters of solvent to preconcentrate analytes from various samples rather than hundreds of millilitres needed in traditional LLE (Sarafraz-Yazdi and Amiri, 2010). One of these techniques, single-drop microextraction (SDME) (Liu and Dasgupta, 1995) has drawn much interest. Recently, several review papers have addressed this emerging technique as an efficient approach to the pretreatment in a wide variety of analytes and matrices (Jain and Verma, 2020, Jeannot et al., 2010; Kumar Kailasa and Wu, 2013; Tang et al., 2018; Tegladza et al., 2020, Zichová et al., 2018a) highlighting the green approach of the technique in minimisation of the hazards associated with the use of toxic organic solvents.

The principle of SDME is the separation of compounds between a few microlitres volume drop of organic solvent, named microdrop, at the tip of a microsyringe needle or in the headspace above the sample and a liquid sample containing the desired analytes. The syringe needle is immersed into the sample and the microdrop of solvent is exposed to the sample for limited time at specified conditions. SDME is often used to represent the acceptor phase where only a small microdroplet $(0.5-2.0 \mu \mathrm{L})$ is suspended on the tip of a microsyringe. After sampling/extraction of analytes, the microdrop is drawn into the syringe and all its volume is analysed by an instrumental analytical technique such as gas chromatography with the appropriate detection. Development of an analytical method using SDME requires optimisation of a high number of variables and parameters affecting the extraction step. It has been shown in our previously published review (Zichová et al., 2018b) that parameters such as the type of investigated analytes, quality of the extraction solvent, type of microsyringe, sample volume, physical properties such as temperature, ion strength and $\mathrm{pH}$ of the sample affect the efficiency of the extraction procedure. It was stated that in SDME development, it is important to find extraction conditions ensuring drop stability. The ease of dislodgment of the microdrop hanging from the tip of the microsyringe needle during the extraction process limits the use of extended extraction times, high stirring rates, sample temperature and the type of sample matrix (Jain and Verma, 2011).

Only a few comprehensive information on the influence of the liquid sample matrix on the stability 
and hold-up of the microdrop hanging from the tip of the microsyringe needle during the extraction process are available. Therefore, the aim of this paper was to investigate the microdrop hold-up in SDME for four various liquid samples, namely orange juice, alcohol-containing sample, deionised water and milk. The influence of SDME parameters on the food analysis application area was studied.

\section{Materials and Methods}

\section{Chemicals}

Several organic solvents were used for microdrop stability evaluation. Chloroform and acetone were purchased from Centralchem, s.r.o. (Bratislava, Slovakia). Dichloromethane, $n$-hexane, tetrachloromethane, and toluene were purchased from Merck KGaA (Darmstadt, Germany). Chlorobenzene was obtained from Lachema, n.p. (Brno, Czech Rep.) and tetrachloroethane from Alfa Aessar (Havehill, USA). Ion strength of the sample was altered by $\mathrm{NaCl}$ obtained from laboratory sources.

\section{Samples}

Orange juice (100\%), plum distillate (40\% alc.), and milk $(0.5 \%$ of fat) were purchased from local shops. These samples were diluted with deionised water and used for the extraction as follows: Orange juice was diluted in the ratio $1: 2(\mathrm{v} / \mathrm{v})$ and $1: 10(\mathrm{v} / \mathrm{v})$, alcohol samples were each diluted to $20 \%$ alc., and milk was diluted in the ratio 1:10 (v/v). The sample volume of $2 \mathrm{~mL}$ for each sample was used in the experiments. Grape distillate (62\% alc.) was obtained from a private producer.

\section{Equipment}

For the extraction procedure, a $10 \mu \mathrm{L}$ Agilent Technologies microsyringe was used to maintain the microdrop during the extraction and to determine the microdrop volume. A glass vial containing the sample solution, magnetic stir bar and silicone septum was used as the sample reservoir.

Magnetic stirrer IKA RTC basic safety control (IKA $\left.{ }^{\circledR}\right)$ with adjustable speed range from 50 to $1500 \mathrm{rpm}$, digital display and aluminium heating plate was used to stir the sample solution. A scheme of SDME is shown in Fig. 1.

\section{Results and Discussion}

Stability and hold-up of the microdrop at the tip of the microsyringe needle in SDME is affected by many parameters. The primary concern of the study was to investigate the matrix influence on the stability of the microdrop on the tip of the microsyringe needle for direct immersion (DI) and headspace (HS) mode of SDME. Several matrices were investigated to cover a large scale of water-based matrices with various organic solvents and stirring conditions. Specifically, microdrop stability for acids-containing matrix (orange juice), alcohol-containing matrix (plum distillate, grape distillate), water matrix and protein-containing matrix (milk) was studied.

\section{Single drop microextraction procedure}

Microsyringe was rinsed six times with acetone, then six times with $n$-hexane and three times with the tested extraction solvent. The plunger was placed in position 0 and the tested extraction solvent (volumes in the range of $1-6 \mu \mathrm{L}$ ) was drawn into the syringe. Subsequently, the microsyringe needle was inserted through the silicon septum and immersed in the sample solution (volume of the sample was $2 \mathrm{~mL}$ ) under stirring (in the range of 100-1000 rpm). After the extraction time (tested range: 5-20 min) passed, the microdrop was drawn back into the microsyringe and the needle was removed from the glass vial. The volume after the extraction was registered. Each ex-

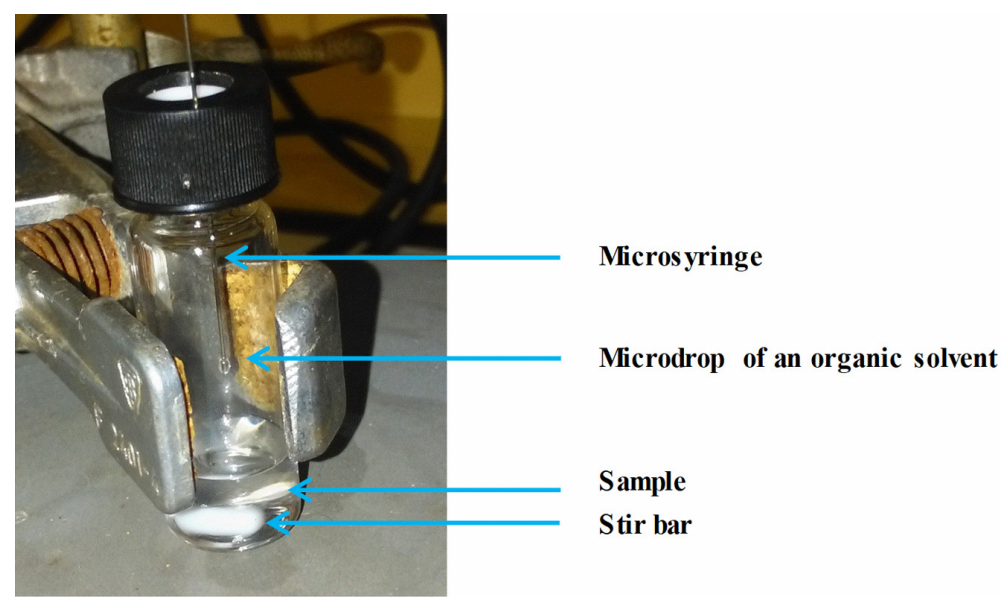

Fig. 1. Scheme of SDME. 
periment was repeated three times. Figs. 1-4 show the average volume of the extraction solvent after the extraction.

\section{Microdrop behaviour in water}

Stability of the microdrop was investigated in a simple matrix - deionised water, assuming no matrix components affecting the microdrop stability. The selection of organic solvent is a crucial step in ensuring good performance of SDME. The selected solvent has to preserve the integrity of the microdrop during the extraction. For microdrop extraction in an aqueous-based solution by DI, immiscibility of the organic solvent in the sample has to be ensured. Also viscosity, which should be adequate for the formation and retention of the microdrop on the tip of the microsyringe needle during the extraction of the SDME process, has to be considered in the extractant solvent selection. Several chlorinated solvents such as tetrachloromethane (TCM), chloroform (CHF), dichloromethane (DCM), tetrachloroethane (TCE) and chlorobenzene (CHB) meet these requirements and they were used for microdrop formation. A volume of $2 \mathrm{~mL}$ of deionised water was stirred for $2 \mathrm{~min}$ at $100 \mathrm{rpm}$ and $250 \mathrm{rpm}$, and the volume of $2 \mu \mathrm{L}$ of the studied solvent was exposed to the stirred sample. After the selected period of time, the volume of the withdrawn extraction solvent was recorded. All of the studied extraction solvent microdrops were stable in the water sample, except for the DCM microdrop, which fell off the syringe needle after half a minute at $100 \mathrm{rpm}$. Volumes above $1.8 \mu \mathrm{L}$ at both $100 \mathrm{rpm}$ and $250 \mathrm{rpm}$ were withdrawn using solvents CHB, TCE and TCM, whereas CHF microdrop could not be withdrawn back into the microsyringe without air bubbles, which complicated the determination of the withdrawn volume. The decrease of the CHF microdrop volume of more than $25 \%$ was observed at higher stirring speed.

\section{Microdrop behaviour in orange juice}

Orange juice is a complex matrix which contains, apart from acids and sugars, also pigments. Organic acids can alter the sample $\mathrm{pH}$ and its initial check is thus necessary. Pigments can easily transfer into the microdrop and result in its dislocation from the tip needle. Also, high content of fibres is a problem and therefore dilution or preconcentration of the sample were tested. Considering these requirements based on our previously published literature review (Zichová et al., 2018 b), toluene is the first choice as solvent for these samples. Therefore, toluene was tested as the extractive solvent, showing immiscibility with the sample with non-colour microdrop after extraction. Stability of the toluene microdrop for HS and DI depends on the stirring rate as well as on the volume of microdrop drawn back to the microsyringe after its extraction and dislocation were evaluated. The stirring rate has to be investigated considering the microdrop integrity. Higher agitation rates increase the occurrence of microdrop displacement from the microsyringe tip and decrease the volume of the extractant after the extraction due to the dissolution of the solvent microdrop in the sample.

Experimental assays were performed using concentrated original orange juice, orange juice diluted with water in the ratio of 1:10 $(\mathrm{v} / \mathrm{v})$ and in that of 1:2 (v/v). Influence of the sample dilution on the microdrop stability was also studied. The results were recorded and compared. All sets of measurements were performed at various stirring rates; the maximum stirring rate depended on the microdrop hold-up at the microsyringe tip. Data showing stability of the toluene microdrop in DI mode for all tested samples: diluted juice sample in the ratio of $1: 10$, diluted sample in the ratio of $1: 2$ and concentrated original sample, and in dependence on the stirring rate are depicted in Figs. 2A, 2B and 2C, respectively. The column bars represent the volume of extractant originally taken for the extraction and the number at the top of the column bar represents the volume of toluene measured after the extraction. The toluene volume after the extraction equal to 0 means that the microdrop was dislocated during the experiment and therefore no toluene was drawn back to the microsyringe.

As it can be seen in Fig. 2A, the widest range of toluene volume $(1-6 \mu \mathrm{L})$ was determined in orange juice sample diluted in the ratio of 1:10. The microdrop was easily visible despite the sample colouring and the stability was notable in the whole range of the studied volumes for lower stirring rates in all experiments (100 rpm and $250 \mathrm{rpm})$. Higher stirring rates $(>500 \mathrm{rpm}$ ) caused instability of microdrop with the volume higher than $5.5 \mu \mathrm{L}$ at the stirring rate of $500 \mathrm{rpm}$ and that with the volume higher than $1 \mu \mathrm{L}$ at the stirring rate of $600 \mathrm{rpm}$.

Further experiments with sample diluted in the ratio of 1:2 and the un-diluted sample showed differences in the maximum microdrop volume as well as conditions at which the microdrop was stable. Stirring rate of $750 \mathrm{rpm}$ was excluded from the experiments due to the microdrop instability; the microdrop up to the volume of $2.5 \mu \mathrm{L}$ was stable at stirring rates up to $500 \mathrm{rpm}$. However, in both cases, visibility of the microdrop in the sample was low, and an effort to white-out the samples was made in un-diluted sample. Nevertheless, the microdrop was invisible even when a lightening compound was applied.. Thus, withdrawal of the microdrop after extraction is complicated. 


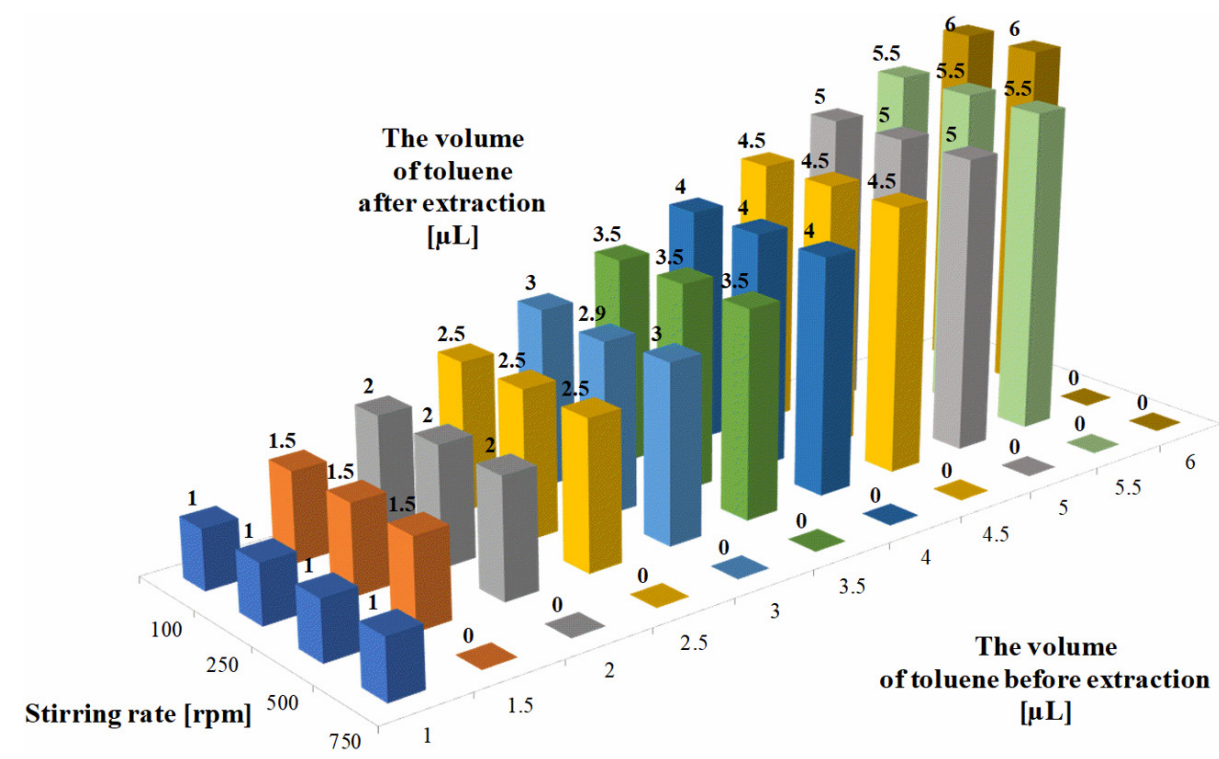

A

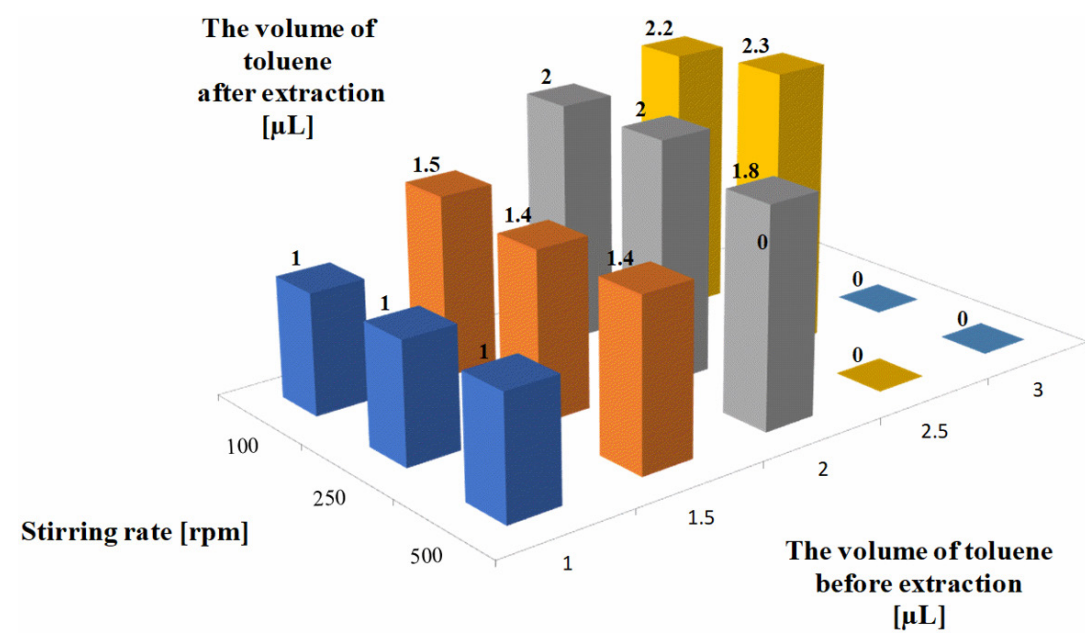

B

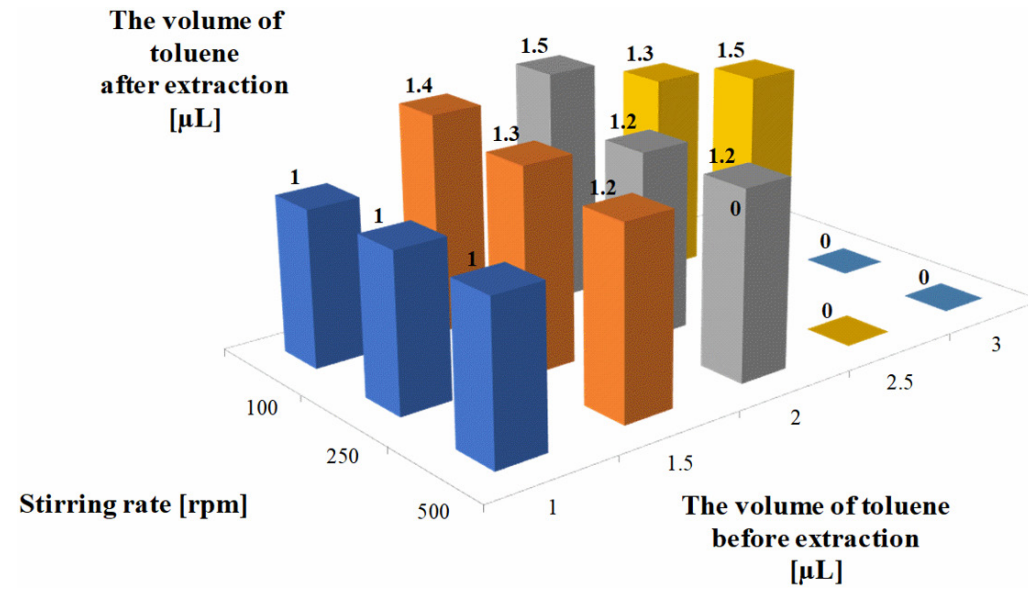

C

Fig. 2. Stability of toluene microdrop (DI-SDME) at various stirring rates in: A - orange juice sample diluted with water in the ratio of $1: 10 ; \mathrm{B}$ - orange juice sample diluted with water in the ratio of 1:2; $\mathrm{C}$ - original orange juice sample (undiluted). 
The stability of toluene microdrop was investigated in the HS mode in the range of $1-4 \mu \mathrm{L}$, stable microdrop was observed up to $3 \mu \mathrm{L}$ for all sample types and at stirring rates between $500 \mathrm{rpm}$ and $1400 \mathrm{rpm}$. It is important to emphasize that the difference in the volume before and after extraction was higher for the DI mode than for HS mode. In addition, the decrement of the microdrop volume in the DI mode increased in the order: diluted sample in the ratio of $1: 10<$ diluted sample in the ratio of $1: 2<$ un-diluted sample.

Microdrop behaviour in alcohol-containing samples Stability of toluene microdrop in the sample with alcohol content of above $40 \%$ was studied. Plum distillate with the alcohol content of $40 \%$ and grape distillate with the alcohol content of $62 \%$ were used in these experiments. These samples were diluted with deionised water in order to obtain a $20 \%$ alcohol content of each distillate and results from experiments using samples with higher alcohol content were compared with those with lower alcohol content. Detailed results showing the stability of toluene microdrop in the DI mode for plum distillate with the alcohol content of $40 \%$ and $20 \%$ are depicted in Figs. 3A and 3B, respectively.

Microdrop volumes ranging from $1 \mu \mathrm{L}$ to $4 \mu \mathrm{L}$ were examined in case of the $40 \%$ plum distillate and in the range of $1-6 \mu \mathrm{L}$ for the $20 \%$ plum distillate. Observations showed that higher alcohol content limited the microdrop volume (limit was $3 \mu \mathrm{L}$ ) and stirring rates (limit was $750 \mathrm{rpm}$ ). A $3 \mu \mathrm{L}$ toluene mi-

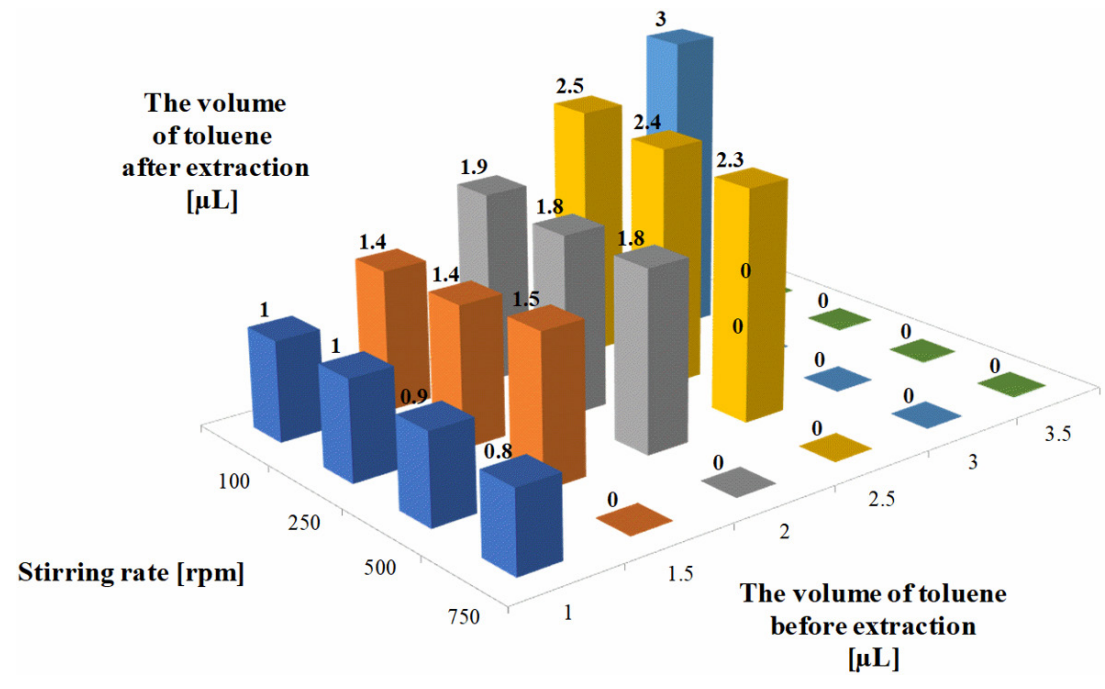

A

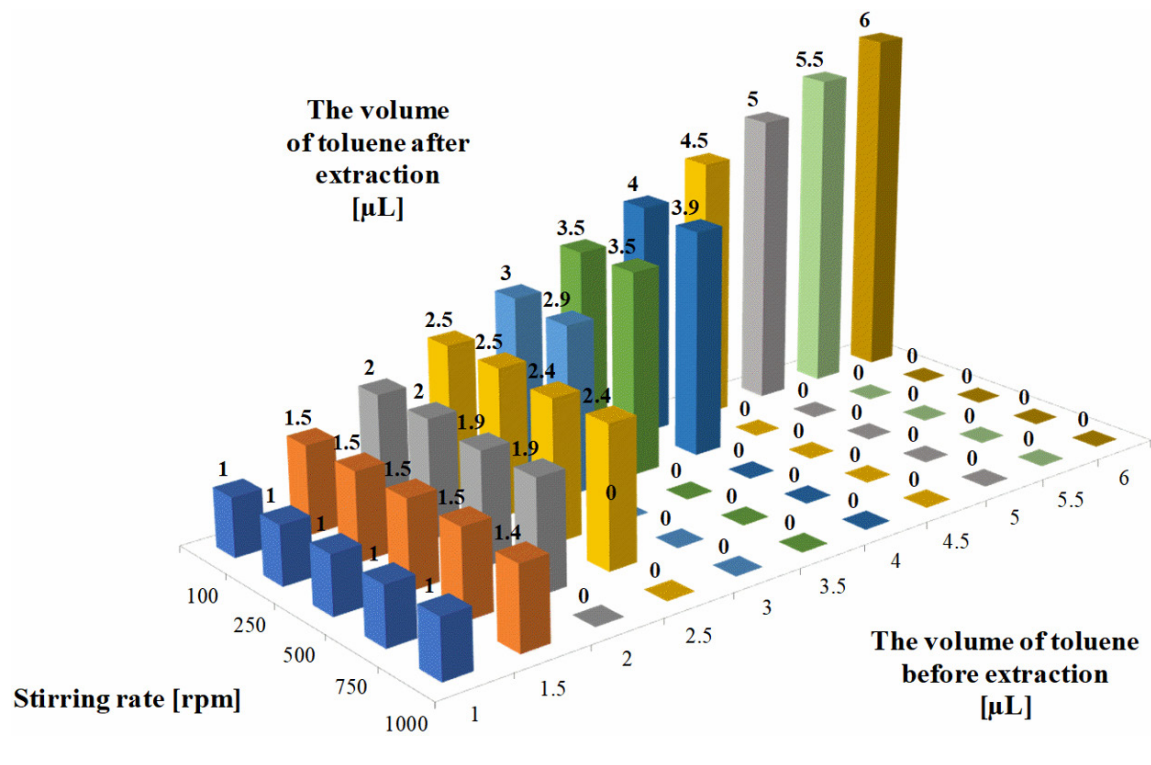

B

Fig. 3. Stability of toluene microdrop (DI-SDME) at various stirring rates in: A - plum distillate $40 \%$; $\mathrm{B}$ - plum distillate $20 \%$. 
crodrop was stable using the lowest studied stirring speed of $100 \mathrm{rpm}$ and no notable decrement of the extractive solvent was observed. At higher stirring speeds, a $3 \mu \mathrm{L}$ microdrop was unstable at the tip of the needle and dropped off after a couple of seconds of stirring. Dilution of the plum distillate sample to half the alcohol content with deionised water enabled using considerably higher toluene microdrops (up to $6 \mu \mathrm{L}$ ) and higher stirring rates (up to $1000 \mathrm{rpm}$ ). The microdrops of all tested volumes were stable at the lowest stirring rate of $100 \mathrm{rpm}$, whereas those with the volume of up to $2.5 \mu \mathrm{L}$ were stable up to the stirring rate of $750 \mathrm{rpm}$. Lower microdrop volumes of up to $1.5 \mu \mathrm{L}$ led to microdrop instability at the highest stirring rate $(1000 \mathrm{rpm})$.

Similar results were obtained in experiments using the $62 \%$ grape distillate, when the toluene microdrop showed stability difficulties even at low stirring rates $(100 \mathrm{rpm}, 200 \mathrm{rpm})$. The maximal stable microdrop volume was about $1.5 \mu \mathrm{L}$ at $100 \mathrm{rpm}$. Presumably, high alcohol content caused a significant decrement of the toluene microdrop after the extraction probably due to the toluene solubility in ethanol (dos Anjos et al. 2015). Dilution of the grape sample to $20 \%$, significantly improved the microdrop stability similarly as in case of the plum distillate, while the toluene microdrop was stable up to the volume of $6 \mu \mathrm{L}$.

It is necessary to consider the change of alcohol content in real-life samples and to make the respective correction or fix the alcohol content to constant value.

\section{Microdrop behaviour in milk samples}

Due to high content of proteins and lipids in milk and their solubility in non-polar solvents such as toluene, an appropriate extraction solvent with the lowest possible solubility in milk ensuring stable microdrop during the extraction has first to be identified. The stability of toluene microdrop in the milk sample (DI mode) was studied at the stirring rates of $100 \mathrm{rpm}$ and $250 \mathrm{rpm}$, and the extraction time varied from 5 min to $25 \mathrm{~min}$ (with a 5 -minute increment for consecutive experiments). The milk sample was diluted with deionised water in the ratio of 1:10 (v/v) and the aliquot volume of $2 \mathrm{~mL}$ was used in the experiments. Results showed stability problems of the toluene microdrop at the stirring rate of $250 \mathrm{rpm}$. The main limitation of toluene application was the loss of the microdrop volume after the extraction exceeding $25 \%$ of the original microdrop volume. At high extraction times (e. g. $20 \mathrm{~min}$ ), microdrop volume after the extraction was below $1.7 \mu \mathrm{L}$ (original volume was $2 \mu \mathrm{L}$ ). Based on the results, satisfactory microdrop stability was obtained under the following extraction conditions: extraction time of $5 \mathrm{~min}$ and sample stirring rate of $100 \mathrm{rpm}$. However, due to the short extraction time of $5 \mathrm{~min}$, several different extractive solvents were studied.

A set of experimental assays using TCM, CHF, DCM, TCE and CHB as extractive solvents were performed with a milk sample diluted with deionised water in the ratio of 1:10 (v/v). The sample volume was set to $2 \mathrm{~mL}$. Significant difference in the stability of the above-mentioned chlorinated solvents was observed and only the CHB microdrop was stable in the diluted milk sample. Microdrops of other solvents fell down immediately after the microdrop exposure to the sample. Thus, subsequent experiments were devoted to the CHB microdrop stability. Extraction parameters were as follows: stir-

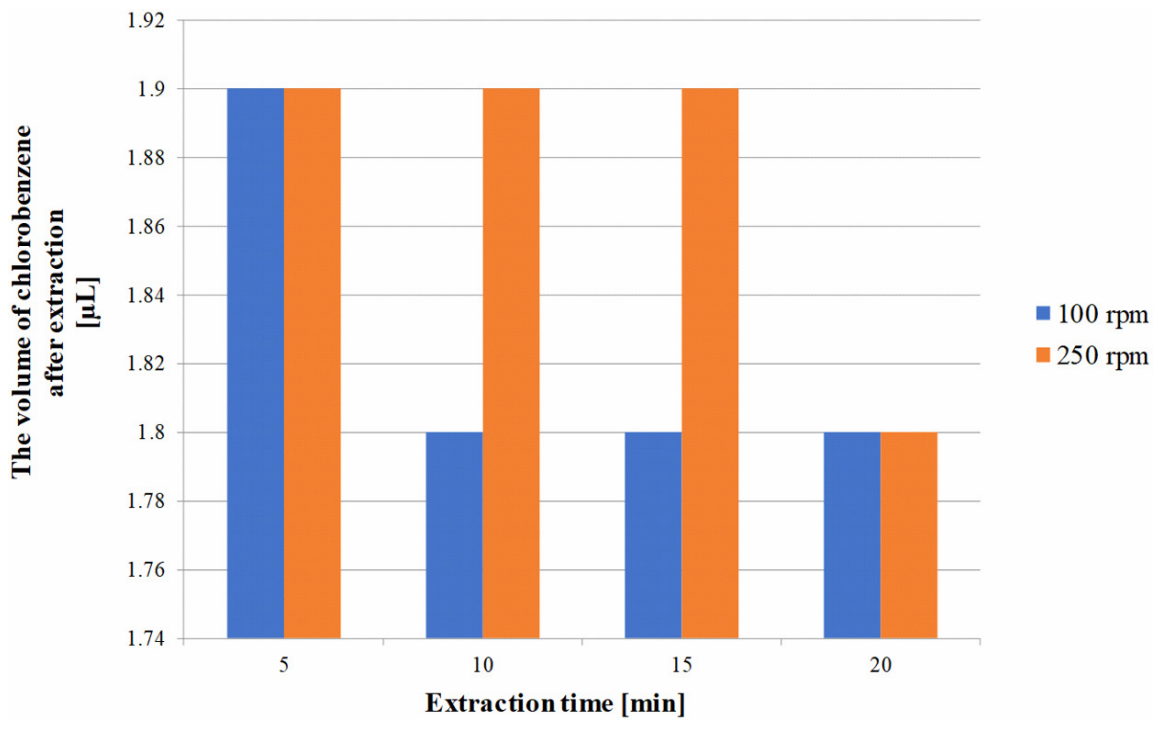

Fig. 4. Dependence of CHB microdrop volume after DI-SDME at the stirring speed of $100 \mathrm{rpm}$ and $200 \mathrm{rpm}$ on the extraction time. 
ring rates of $100 \mathrm{rpm}$ and $250 \mathrm{rpm}$, extraction time in the range of 5 min to 25 min (with a 5 -min step). The disadvantage of the DI mode is the impossible visual control of the microdrop in the sample which could lead to a delay in sample treatment if the microdrop fell down from the needle tip. Moreover, differences in the microdrop volumes withdrawn after the extraction in the three samples were notable due to the worse visibility of the microdrop. The maximum CHB microdrop volume withdrawn in the dependence on extraction time is shown in Fig. 4. Relative standard deviation (RSD) for different combinations of stirring rate and extraction time was in the range of $3.1-6.9 \%$. Based on these observations, sample centrifugation and the addition of salt to the sample were tested to increase the possibility of visual control in the sample.

The diluted milk sample was centrifuged at $4000 \mathrm{rpm}$ for $5 \mathrm{~min}$. CHB microdrop exhibited lower stability at both stirrings rates and the centrifugation did not improve the microdrop visibility. In the next step, the addition of $\mathrm{NaCl}$ to the diluted milk sample after centrifugation was studied. The $\mathrm{NaCl}$ addition was carried out successively in the range of $0.1-0.4 \mathrm{~g}$ (with a step of $0.1 \mathrm{~g}$ ). The sample was clarified by the salt addition and the drop was easily observed. Furthermore, salt addition improved the stability of microdrop directly immersed in the sample. Increasing the amount of $\mathrm{NaCl}$ consecutively improved the stability of the CHB microdrop at both stirring rates and at all tested extraction times. The most satisfactory microdrop stability was obtained at the salt addition of $0.4 \mathrm{~g}$. Lower $\mathrm{NaCl}$ addition led to the instability of the microdrop after $15 \mathrm{~min}$ as well as a decrement of the extracted volume (withdrawn volume was $1.7 \mu \mathrm{L}$ ).

\section{Conclusions}

SDME is a sample preparation technique for various instrumental detection analyses employing simple equipment and leading to low environmental impact. The essential condition of SDME is maintaining a microdrop of an organic solvent suspended at the tip of a microsyringe needle, which is then immersed in a sample under agitation. Drop instability and its dislocation are the main disadvantages of this technique. The microdrop has to be retracted back into the microsyringe and transferred to the subsequent system for analyses system and thus detailed optimisation of conditions such as solvent type, solvent volume, agitation type and time etc. is crucial. The change of matrix composition (content of acid compounds, content of alcohol, protein content) has been proven to affect the stability of the solvent microdrop. Concentration of fibres and organic acids also affects the microdrop solubility and stability. For alcohol content samples, the content of alcohol has to be considered in solvent selection because of the solubility either of microdrop in water or in alcohol; the alcohol content should be determine before the analysis. For milk samples, careful optimisation of extraction parameters has to be done in dependence on protein and fat content, or the removed matrix has to be tested.

\section{Acknowledgement}

This work was supported by the Scientific Grant Agency of the Slovak Republic VEGA project No. 1/0412/20 and the Program for support of excellent teams of young researchers $S T U$. NK would like to thank for the kind support of AXA Foundation and the foundation of Pontis in the frame of the "Young innovator" program.

\section{References}

Andraščíková M, Matisová E, Hrouzková S (2015) Sep. Purif. Revs. 44: 1-18.

dos Anjos JP, de Andrade JB (2015) Microchem J. 120: $69-76$.

Hrouzková S (2017) In: "Food analysis: Innovative analytical tools for safety and quality assessment", Umile Gianfranco Spizzirri, Giuseppe Cirillo (Ed), John Wiley \&Sons, Hoboken, New Jersey and Scrivener Publishing, Beverly, MA, p. 221.

Jain A, Verma KK (2011) Anal. Chim. Acta 706: 37-65.

Jain A, Verma KK (2020) In "Liquid-Phase Extraction". Poole CF (Ed), Elsevier, London UK, pp. 439-472.

Jeannot MA, Przyjazny A, Kokosa JM (2010) J. Chromatogr. A 1217: 2326-2336.

Kumar Kailasa S, Wu H-F (2013) Bioanalysis 5 (21): 2593-2596.

Liu S, Dasgupta PK (1995) Anal. Chem. 67: 2042-2049.

Sarafraz-Yazdi A, Amiri A (2010) Trends in Anal. Chem. 29: 1-14.

Tang S, Qi T, Ansah PD, Nalouzebi Fouemina JCh, Shen W, Basheer Ch, Lee HK (2018) Trends in Anal. Chem. 108: 306-313.

Tegladza ID, Qi T, Chen T, Alorku K, Tang S, Shen W, Kong D, Yuan A, Liu J, Lee HK (2020) J. Hazard. Mat. 393 No 122403.

Zichová S, Brisudová A, Hrouzková S (2018a) Nova Biotechnol Chim 17(1): 1-15.

Zichová S, Brisudová A, Hrouzková S (2018b) Acta Chimica Slovaca 11: 60-67. 